\title{
Expression of Interest
}

\section{A Muon to Electron Conversion Experiment at Fermilab}

\section{The mu2e Collaboration}

E.J. Prebys*, D. Bogert, D.R. Broemmelsiek, C.M. Ankenbrandt, S.J. Brice, D.F. DeJongh, S. Geer, D.E. Johnson, M.A. Martens, D.V. Neuffer, M. Popovic, R.E. Ray, K. Yonehara, H.B. White, C.Y. Yoshikawa

Fermi National Accelerator Laboratory

J.P. Miller*, R.M. Carey, K.R. Lynch, B.L. Roberts

Boston University

P. Yamin, M.V. Diwan, W.J. Marciano, Y. Semertzidis

Brookhaven National Laboratory

Yu.G. Kolomensky

University of California, Berkeley

J.L. Popp, D. Dale, K.J. Keeter, E. Tatar

Idaho State University

P. Kammel, P.T. Debevec, D.W. Hertzog

University of Illinois, Urbana-Champaign

V. Lobashev

Institute for Nuclear Research, Moscow, Russia

K.S. Kumar, D.M. Kawall

University of Massachusetts, Amherst

T.J. Roberts, R.J. Abrams, M.A.C. Cummings, R.P. Johnson, S.A. Kahn, S.A. Korenev, R.C. Sah Muons, Inc.

P.A. Souder, R.S. Holmes

Syracuse University

E.C. Dukes, M.A. Bychkov, E. Frlez, R.J. Hirosky, A.J. Norman, K.D. Paschke, D. Pocanic University of Virginia

*Co-Contact Person 


\section{Expression of Interest}

\section{A Muon to Electron Conversion Experiment at Fermilab}

We are writing this letter to express our interest in pursuing an experiment at Fermilab to search for neutrinoless conversion of muons into electrons in the field of a nucleus, which is a lepton flavorviolating $(L F V)$ reaction. The sensitivity goal of this experiment, improving on existing limits for this process by more than a factor of 10000, is very similar to that of previous experiments that have been proposed but never built. It would provide the most sensitive test of LFV, a unique and essential window on new physics unavailable at the high energy frontier. We present a conceptual scheme that would exploit the existing Accumulator and Debuncher rings to generate the required characteristics of the primary proton beam. The proposal requires only modest modifications to the accelerator complex after including those already planned for the NOvA experiment, with which this experiment would be fully compatible.

\section{Introduction and Motivation}

The search for lepton flavor violation (LFV) has long played an important role in the evolution of our understanding of electroweak interactions. The neutrinoless conversion of a muon to an electron in the field of a nucleus is a particularly interesting example of an LFV process involving charged leptons. In the Standard Model, such conversions would take place via loop diagrams involving virtual neutrino mixing, at a rate far below the threshold of any currently conceivable experiment. Indeed, any detectable signal would be a definite indication, albeit indirect, of new dynamics at multi-TeV energy scales. Enhanced rate for this process is an almost universal feature of beyond the Standard Model physics, and the fact that such a process has not been observed has constrained or eliminated many models [1].

While it is widely believed that new physics will appear at LHC energies, the LHC is not well-equipped to study LFV directly. An often-quoted example is in the case of supersymmetry. The LHC will probe slepton masses, but it cannot compete with muon decay experiments in constraining the slepton mixing angles. Sensitive searches for rare or forbidden leptonic and semi-leptonic LFV processes, especially those involving charged leptons, are essential for the comprehensive characterization of new high energy physics.

While there are several potential reactions that can be used to probe LFV, muon to electron conversion has the remarkable feature that it does not require the coincidence of two final-state particles. The spectacular signature is a single conversion electron of well-defined energy, separated from most of the sources of background. As a result, very high muon data rates can be handled and an unusually sensitive search for LFV becomes feasible. Indeed, at the level of sensitivity discussed below, a large class of supersymmetric models would predict 100's of conversion events. Additionally, compositeness and Z' models would be probed at the multi-TeV scale in a manner complementary to direct LHC searches. Lepto-quarks would be probed at the $3000 \mathrm{TeV}$ scale. Muon to electron conversion is therefore sensitive to many new physics scenarios at energy scales that cannot be probed by direct searches using other foreseeable accelerators. 


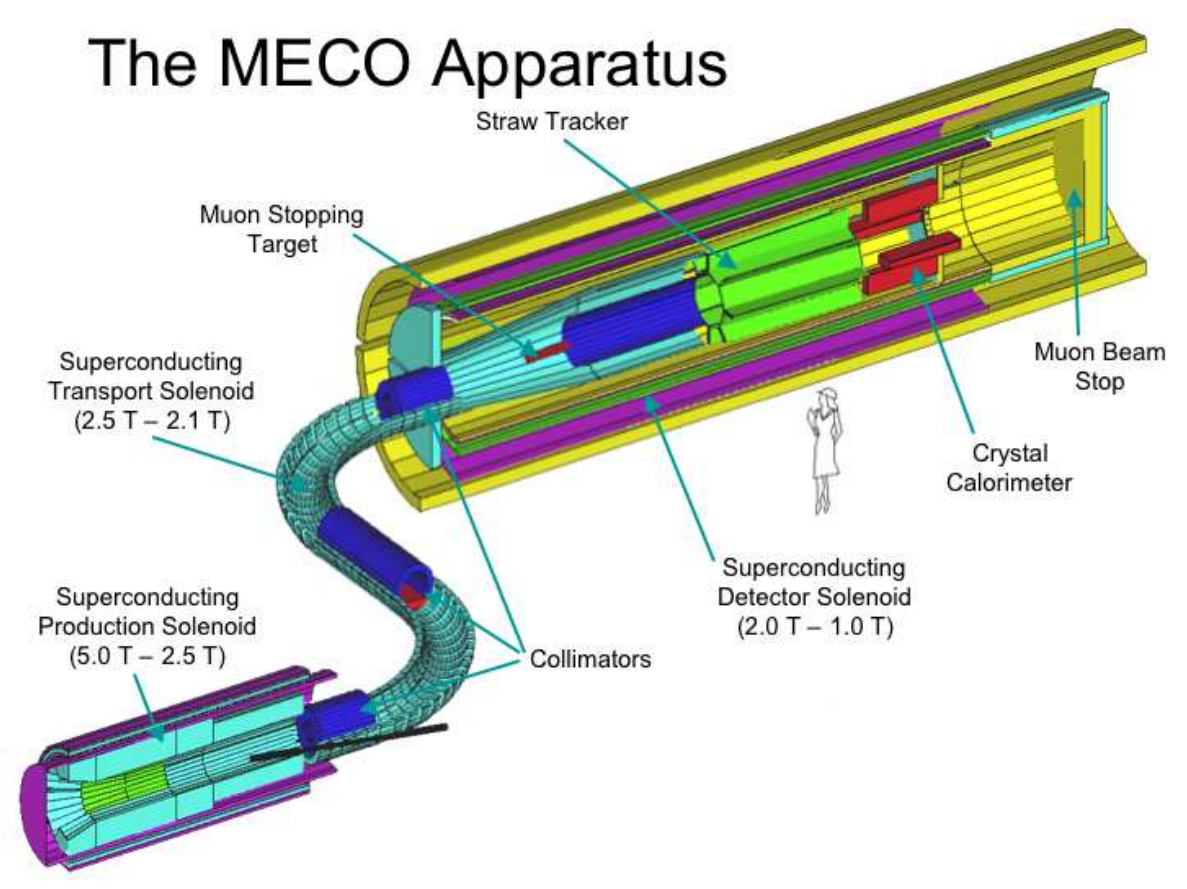

Figure 1: Layout of the proposed MECO experiment.

\section{Experimental Apparatus}

An ambitious experiment to study this physics was proposed for the AGS at Brookhaven. Called the Muon to Electron COnversion (MECO) experiment, it was based on a muon beam line concept that was first proposed for the MELC experiment [3] at the Russian Institute for Nuclear Research (INR) a few years earlier. The MECO experiment was designed to search for the signature of a captured muon converting to an electron through the exchange of virtual particles with an Aluminum nucleus. The MECO experiment is extensively documented in its technical proposal [2], and the physics case is described in detail in the first chapter of that document. The experimental layout is shown in Figure 1.

The main components are a proton target and a sophisticated solenoidal channel, which transports low energy muons to an Aluminum stopping target. This target is surrounded by a detector geometry optimized to detect the monoenergetic electron produced in the conversion process, and to have very low acceptance for the large electron background at half the muon rest energy and below. The detector design is based on the experience gained from the SINDRUM muon to electron conversion experiment which was conducted at PSI in the 1990's, and which established the current experimental limit on this process.

A key feature of the experiment is the beam bunch structure. Protons are delivered to the target in short ( $\sim 100 \mathrm{~ns})$ bunches with a separation on the order of the muon lifetime ( $1 \mu \mathrm{sec}$ for a muon captured by Aluminum). Conversion electrons must be detected between bunches. In order to reduce false signal background, the suppression of out of bunch protons, or "extinction factor", is of paramount importance, and the MECO experiment was designed to achieve an extinction factor of $10^{9}$. For a total of $4 \times 10^{20}$ protons delivered, this would have given a single event sensitivity corresponding to a branching ratio of 
$2 \times 10^{-17}$, representing an improvement of more than four orders of magnitude compared to the existing limit $^{1}$.

MECO, together with KOPIO (a search for $K^{0} \rightarrow \pi^{0} v \bar{\nu}$ ), was part of the RSVP project to be performed at the Brookhaven AGS; however, the NSF took the decision not to fund it. Neither the physics importance nor the technical feasibility of MECO was ever in question. Indeed, the last two reviews in June 2005 were extremely positive [6]. There were a number of factors that led to the cancellation of RSVP, and most were related to cost issues. For example, the cost to refurbish the AGS to provide appropriate beam to each experiment was unexpectedly high. Also, the cost of the experiments had risen. (In the case of MECO, this was primarily due to increases in the estimated cost of the magnet system.) Finally, there were uncertainties about the sharing of out-year AGS operating costs between the NSF and DOE/NP.

Because the physics case remains compelling, we have been investigating the practicality of mounting a similar experiment (herein called the "mu2e" experiment) at Fermilab in the era following the termination of the collider program. Our initial look shows that it is highly feasible and meshes well with the neutrino program.

Prior to its cancellation, MECO had passed through many major reviews, technical and fiscal, all with positive results, and represents an advanced conceptual design. Although there may have been developments in the last few years that could enable us to improve the sensitivity of a muon-to-electron conversion experiment, we will assume for the moment that such an experiment would be more or less identical to MECO and we will focus instead on the feasibility of delivering a suitable beam. We consider it axiomatic that our experiment would have to coexist with the NOvA experiment.

\section{Proton Beam Line}

We propose to develop an appropriate beam line at FNAL. The work accomplished to date is described in more detail elsewhere [7], of which a schematic illustration is shown in Figure 2. In terms of total protons, the existing Proton Source should have enough excess capacity to provide for the mu2e experiment even with the increased intensity planned for the NOvA program.

The challenge will be to provide the desired beam structure. This problem can be solved by re-tasking the $8 \mathrm{GeV}$ storage rings (the antiproton Debuncher and Accumulator) as depicted in the detail of Figure 2. In current operation, antiprotons are transported from the production target into the outer Debuncher ring, where they are phase rotated and stochastically cooled. They are then transferred into the inner Accumulator ring, where they are "momentum stacked" onto the core and further cooled.

Our proposed scheme would reverse the order. Booster proton batches would be injected directly into the Accumulator, where several would be momentum stacked. These would then be transferred into the Debuncher ring and rebunched into a single, short bunch. Finally, the beam would be resonantly extracted, such that this single bunch would become a bunch train. The $1.7 \mu$ sec period of the Debuncher would produce a structure almost ideally suited to the mu2e experiment.

\footnotetext{
${ }^{1}$ The SINDRUM II experiment has the lowest published limit of $4.3 \times 10^{-12}$ (90\% CL) for $\mu \mathrm{Ti} \rightarrow \mathrm{e}$ Ti[4], but we base our comparison on the lower limit of $<6.1 \times 10^{-13}(90 \% \mathrm{CL})$ which they have reported at conferences[5].
} 


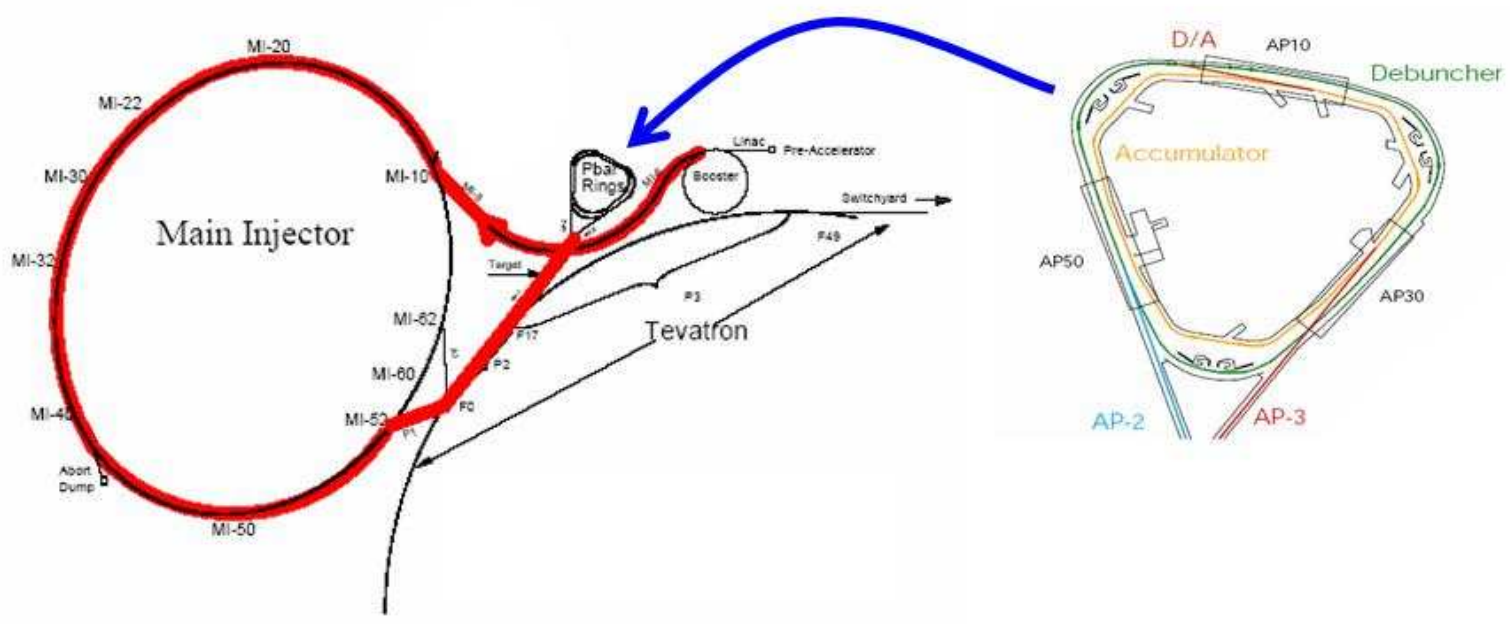

Figure 2: The relevant parts of the accelerator complex are shown. At right is a detail of the antiproton ring, showing the Debuncher (outer ring) and Accumulator (inner ring). The complete path taken by the protons in the proposed "boomerang scheme" is shown at left.

Initially, we believed that the need to inject protons into the Accumulator during NOvA running would require a new transport line to be built from the Booster to the Debuncher/Accumulator enclosure. However, an ingenious "boomerang scheme" has been proposed which would allow Booster beam to be transported to the Accumulator with no civil construction and indeed minimal beam line modifications of any kind.

A key part of the NOvA accelerator upgrades will be a modification to the MI- 8 line to allow beam to be directly injected into the Recycler. Booster batches will be slip stacked in the Recycler prior to being loaded into the Main Injector. This will eliminate the time currently spent loading the Main Injector and increase the total proton rate to the NuMI line. In the NOvA plan, 12 Booster batches will be stacked into the Recycler during every 1.333 second Main Injector cycle. Because this period corresponds to 20 $15 \mathrm{~Hz}$ Booster cycles, there are potentially up to eight extra batches available.

In order to take advantage of this unused part of the time line, we would have to add a simple extraction region to the Recycler to direct beam into the existing P150 line. Protons from the Booster would then make only a partial circuit of the Recycler, after which they would be transported to the Accumulator in the same way we currently transport small numbers of "reverse protons" from the Main Injector.

Because the protons don't go all the way around the Recycler, extraction could be done with a simple switched magnet, rather than a kicker. This has the potential to deliver up to 8 Booster batches every 1.33 seconds. If we conservatively assume Booster batches of $4 \times 10^{12}$ protons, this could provide as many as $7.5 \times 10^{20}$ protons per year to this effort - assuming that the total Booster flux could be increased enough to accommodate this ${ }^{2}$. In practice, longitudinal emittance in the Accumulator/Debuncher will probably limit extraction to 4 to 6 batches at a time, or 2.5 to $5.0 \times 10^{20}$ per year ${ }^{3}$.

\footnotetext{
${ }^{2}$ The total Booster output is limited by the maximum average repetition rate that the RF system can support and by radiation issues due to beam loss. Making the Booster robust to $15 \mathrm{~Hz}$ will require at most modest improvements over what is planned
} 
Momentum stacking in the Accumulator is a straightforward modification of what is done now with antiprotons. We are investigating several schemes to arrive at a single short bunch in the Debuncher[8]. One promising example is a hybrid scheme, in which the stacked beam is bunched by an $\mathrm{h}=14 \mathrm{kV} \mathrm{RF}$ system in the Accumulator, then transferred to Debuncher, where it undergoes a 90 degree phase rotation by a $40 \mathrm{kV} \mathrm{h}=1 \mathrm{RF}$ system, followed by an $\mathrm{h}=4$ capture by a $200 \mathrm{kV}$ RF system. Assuming we start with six Booster batches, this results in a single bunch with a 38ns rms length and an energy spread of $\pm 200 \mathrm{MeV}$.

The existing tune working point of the Debuncher is $v_{\mathrm{x}} / \nu_{\mathrm{y}}=9.764 / 9.785$, making the $v_{\mathrm{x}}=29 / 3$ a logical resonance to exploit for slow extraction. Given the low chromaticity of the Debuncher, we can get the beam quite close to the resonance even with the rather large $(\sim 1.5 \%)$ momentum spread resulting from the stacking and rebunching. This means that the resonance could be driven with fairly modest sextupoles. An extraction scheme would be implemented with an electrostatic septum similar to one of the 3 m electrostatic septa ( $80 \mathrm{kV} @ 1 \mathrm{~cm}$ gap) currently used in the Main Injector, placed between the 'Q2 and 'Q3 quadrupoles of the extraction leg. The extraction Lambertson would then be placed after the downstream 'Q0 quadrupole. Because a sharp bend is needed to clear the downstream quadrupole, the logical configuration would be a short Lambertson followed by a $\mathrm{C}$ magnet. An $.8 \mathrm{~T}$ bend field would comfortably clear the downstream quadrupole.

Radiation safety is a critical issue for this project. The Accumulator/Debuncher enclosure was designed for an extremely low flux of antiprotons and so is not heavily shielded. The earth shielding is roughly 13 ' in most outside areas and is 10' or less in the service buildings. Tunnel roof loading limits preclude increasing this overburden, which is more than 10' short of the passive shielding requirements ("Cossairt Criteria") for the proposed intensity. Unlike the Booster, it is practical to fence in the entire area and declare it "Limited Occupancy". This will ameliorate the situation somewhat, but it is still certain we will need some sort of safety system involving interlocked detectors ("chipmunks") of the sort that protects the Booster. It is probable that the services buildings will be locked out entirely while the beam is running at high intensity. Because the documentation needed for the shielding assessment of such a configuration is immense, it is important to begin working on this aspect as soon as possible.

\section{Status and Plans}

We have initiated the formation of a formal "mu2e" collaboration, with the goal of generating a much more complete Letter-of-Intent over the next few months and subsequently a proposal within a year. This collaboration currently consists of scientists and engineers from Fermilab, other laboratories, universities, and the private firm, Muons, Inc. A workshop held at Fermilab in September of 2006 to explore the possibility of such an experiment at Fermilab drew about 50 scientists. New collaborators are being actively recruited.

Cost and resource estimates are in their very early stages, but we can use the work done for the MECO project to set the scale. MECO was very mature and included a detailed Technical Proposal [2] in 2001.

\footnotetext{
for the NOvA project. Radiation will be reduced by improving Booster efficiency through improved orbit control and extraction handling ("beam notching").

${ }^{3}$ These annual proton rates are calculated based on the stated batch sizes and time lines, assuming the canonical "Snowmass Year" of $2 \times 10^{7}$ seconds, and should be used only to set the scale. The Letter of Intent will include more accurate projections.
} 
At a subsequent review in 2005, before which a more detailed cost estimate of the apparatus was carried out, the magnet system was estimated at \$58M and the detector package was estimated at about $\$ 25 \mathrm{M}$. The projected total cost in 2011 dollars would be on the order of $\$ 100 \mathrm{M}$. Because the "boomerang scheme" to deliver the proton beam at FNAL eliminates additional civil construction, the cost of the additional modifications to the Fermilab complex would be small on this scale. Civil construction would of course be required for the experimental beam line and hall. While the siting is in its preliminary stages, a promising location has been identified to the West of the antiproton rings, near the neutrino line of the MiniBooNE experiment. A rough construction time estimate can also be obtained from the MECO design studies. MECO was to be a 5-6 year construction project, driven primarily by the schedule for the design and fabrication of the magnets.

At Fermilab, work is being accomplished by the commitment of some amount of research time primarily by scientists. In order to move forward, we will require some engineering and technical support. Given the advanced status of the design of the equipment downstream of the proton production target, most of the effort will be spent on firming the proton source design. At the same time, we will be mounting an effort to increase the visibility of the project at Fermilab and the funding agencies with the goal of finding support for the Universities to study possible improvements to the MECO design, mainly via simulations, and to continue detector system development and prototyping.

\section{Bibliography}

[1] See, for example, overview talk by W. Marciano (2006) http://mu2e.fnal.gov/selected-documentation/marciano_20060915.pdf

[2] MECO Collaboration, "DRAFT MECO Technical Proposal” (2001) http://www.bnl.gov/npp/mu-e_docs/Draft_MECO_Tech_Prop.pdf

[3] R.M. Djikibaev and V.M. Lobashev, Sov. J. Nucl. Phys. 49(2), (1989) 384; V.S. Abdjev, et al, INR Preprint 786/92 (1992).

[4] SINDRUM II Collaboration, "Test of Lepton Flavor Conservation in $\mu \rightarrow \mathrm{e}$ Conversion on Titanium”, Phys.Lett.B317:631-636,1993.

[5] P. Wintz at the $1^{\text {st }}$ International Symposium on Lepton and Baryon Number Violation, Vancouver (1998).

[6] "HEPAP Subpanel Report on RSVP Project\} (2005) http://www.science.doe.gov/hep/RSVPSubpanelReport.pdf

[7] C. Ankenbrandt, D. Bogert, F. DeJongh, S. Geer, D. McGinnis, D. Neuffer, M. Popovic, E. Prebys, Physics/0611124, FERMILAB-TM-2368_AD-E;

C. Ankenbrandt, D. Harding, D. Johnson, D. McGinnis, M. Popovic, Fermilab BEAMS-DOC2678-V1 (2007)

[8] D. Neuffer, Fermilab BEAMS-DOC-2787-V1 (2007) 